\title{
Emergency surgical tracheotomy in the era of COVID-19 pandemic
}

\author{
Piergiorgio Muriana ${ }^{1, \star}$, Paola Ciriaco ${ }^{1}$, Angelo Carretta ${ }^{1,2}$, Giampiero Negri ${ }^{1,2}$
}

${ }^{1}$ Department of Thoracic Surgery, IRCCS San Raffaele Scientific Institute, Milan, Italy

${ }^{2}$ Vita-Salute San Raffaele University, Milan, Italy

\section{*Correspondence}

muriana.piergiorgio@hsr.it (Piergiorgio Muriana)

\begin{abstract}
At the beginning of 2020 a novel variant of coronavirus, named SARS-CoV-2, was identified as responsible for the development of severe pneumonia and acute respiratory distress syndrome (ARDS) with very high mortality, exceeding 30\%. The disease caused by SARS-CoV-2, called Coronavirus Disease 2019 (COVID-19), was declared a pandemic by WHO on March 11th, 2020. Patients affected by COVID-19 may present with subtle, specific symptoms, but the sudden onset of life-threatening acute respiratory failure is not uncommon. The peculiarities of the disease combined with the single patient's comorbidities, e.g. advanced age and cardiovascular diseases, plus hypoxia and hypotension secondary to ARDS, and multiorgan failure, may lead to unexpected difficulties in the case of tracheal intubation. The occurrence of the 'Cannot-Intubate-Cannot-Oxygenate' (CICO) scenario in COVID-19 patients represents a hazard not only for the patients but also for the assisting healthcare workers due to the high risk of aerosol-generating infected particles during conventional rescue airway procedures. While international consensus guidelines on the management of CICO scenario in COVID-19 patients are still lacking, there is evidence that both scalpel cricothyrotomy (CT) and open surgical tracheotomy (OST) represent valid alternatives for the establishment of a front-of-neck emergency airway. Primary CT requires a staged conversion to formal tracheotomy; conversely, OST represents a definitive mastery of the airway in COVID-19 patients in case of prolonged mechanical ventilation dependency, avoiding a second procedure and further exposure to aerosols. Furthermore, in patients with facial trauma and/or head and neck tumors, OST allows obtaining safe airway control. In the context of the current pandemic, emergency OST procedure in SARS-CoV-2 positives (or with unknown status) requires adequate arrangements and the use of proper personal protective equipment to limit risks for clinicians.
\end{abstract}

\section{Keywords}

Emergency tracheotomy; Open surgical tracheotomy; CICO scenario; Cricothyrotomy; Emergency surgical airway; Endotracheal intubation failure; Tracheostomy; COVID-19; SARS-CoV-2; ARDS

\section{Introduction}

\subsection{COVID-19 pandemic and SARS-CoV-2-related ARDS}

At the end of 2019, a few cases of severe pneumonia of unknown origin were reported among individuals from a live animal market in Wuhan, in the Chinese province of Hubei [1]. A novel variant of beta-coronavirus, named Severe Acute Respiratory Syndrome Coronavirus 2 (SARS-CoV-2) was identified in the bronchoalveolar lavage samples of these patients.

SARS-CoV-2 was proved to be responsible for the development of a large clinical spectrum of diseases, collectively called Coronavirus Disease 2019 (COVID-19), ranging from asymptomatic or subtle symptomatic infections to severe acute distress respiratory syndrome (ARDS). Being the replication of the virus mainly located in the nasopharyngeal tract cells, the respiratory droplets and aerosols from carriers swiftly caused the transmission of the infection worldwide [2]. On March 11th, 2020, COVID-19 was declared a pandemic by the World Health Organization (WHO) [3].

Oxygen therapy delivered by nasal cannula, Venturi mask, or reservoir to maintain an oxygen saturation of hemoglobin over $90 \%$ is the mainstay of treatment at every stage of disease of hospitalized patients with SARS-CoV-2-related pneumonia [4]. Although several molecules, including corticosteroids, antimicrobial and antiviral drugs, immunomodulatory, and anticoagulants, have been used to treat the disease in the context of clinical trials, there is still no specific therapy uniquely approved by international organisms [5]. After the completion of the third phase of clinical trials, a nucleoside-modified RNA 
BNT162b2 vaccine has recently been released, promoting immunization against a membrane-anchored SARS-CoV-2 fulllength spike protein [6]. A massive vaccination campaign is currently running in Italy and many other countries worldwide, aiming at a definitive eradication of the disease.

Despite the proportion of patients requiring hospital admission is relatively low compared to the total number of infected subjects, up to two-thirds of them require intensive care at the onset of respiratory failure [2]. With the pandemic outbreak, therefore, the number of patients with COVID-related severe ARDS progressively increased.

The rate of patients with viral pneumonia and concurrent bacterial infections is variable, ranging from $1 \%$ to $12 \%[7$, 8]. Nevertheless, both community and hospital-acquired coinfections are widely perceived to possibly increase morbidity and mortality in COVID-19 patients. Empirical antibiotic therapy is generally recommended in these patients despite the possibility of multi-drug resistance development. In a study by Cataldo et al. on patients with severe COVID-19 pneumonia in the ICU setting, $49 \%$ of subjects developed a bloodstream infection after a median of 13 days of hospitalization [9]. Fungal infections by Candida spp were evident in about $10 \%$ of patients. Therefore, concomitant bacterial and fungal infections at presentation and during hospitalization may enhance the possibility of unfavorable clinical evolution in patients affected by SARS-CoV-2 related disease.

The mortality rate among patients with ARDS is very high, exceeding 30\%. Despite some authors in the early phase of pandemic asserted that patients affected by COVID-19 might show unique characteristics [10], it was later demonstrated that these patients could be successfully treated with therapeutic approaches similar to those with severe respiratory impairment from other causes [11].

Early recognition of patients at risk for severe clinical evolution is crucial. Several factors have been evaluated for the risk stratification of patients with SARS-CoV-2 infection presenting at the emergency department. Balzanelli et al. showed that gas exchange profile at arterial blood gas (ABG) analysis could predict the presence of pulmonary CT scan alterations in a subgroup of patients presenting with alkalosis, hypoxemia and hypocapnia [12]. This finding was further confirmed by two additional studies reporting that both the incidence of inflammatory infiltrates at chest CT scan and poor prognosis were correlated with higher $\mathrm{pH}$ value, and lower $\mathrm{pCO}_{2}$ and $\mathrm{pO}_{2} / \mathrm{FiO}_{2}$ ratio evaluated at $\mathrm{ABG}$ analysis $[13,14]$.

Among symptomatic patients, $14 \%$ show a severe evolution, and $5 \%$ become critical within two weeks after the onset of the disease. Endotracheal intubation and mechanical ventilation should be considered in presence of refractory hypoxemia, hypercapnia and acidosis, respiratory musculature exhaustion, and hemodynamic instability despite the use of maximal noninvasive respiratory support and pronation [15].

After intubation, most patients are managed with lungprotective ventilation, which involves low tidal volumes and positive end-expiratory pressure (PEEP) rate adequate to guarantee alveolar recruitment [16].

The choice of appropriate timing for endotracheal intubation (ETI) is fundamental not only to obtain better shortand long-term outcomes, but also to avoid potential com- plications related to ETI failure, in particular when facing emergency intubation. In the series presented by Zheng et al., the incidence of unexpected troubles during ETI in 59 SARS-CoV-2 positive patients with respiratory failure has been reported to be $8.5 \%$ [17]. In this study, the rate of unsuccess at first intubation attempt was significant despite the use of a video laryngoscope - ranging between $6.5 \%$ and $16.7 \%$ depending on the tool employed - and the application of rapid sequential induction and intubation protocol by skilled operators. The advanced age of COVID-patients, the high rate of cardiovascular comorbidities, the coexistence of hypoxia and hypotension secondary to ARDS and multiorgan failure at the time of intubation, and the scarce time for the assessment of potentially difficult airway are all critical factors that have been recognized to be responsible for an increased probability of intractable ETI failure $[17,18]$, and therefore, for the need of rescue procedures.

\subsection{The emergency surgical airway (ESA)}

In the emergency setting, the inability to ensure a proper ETI for patient ventilation and oxygenation - the so-called 'CannotIntubate-Cannot-Oxygenate' (CICO) scenario - is one of the factors most affecting short-term prognosis [19]. Although performed by experienced operators, the rate of failed intubations in trauma patients is not negligible. In the study by Ono et al., over $23 \%$ of 537 patients had at least one complication related to ETI, and $5 \%$ of them underwent a rescue emergency surgical airway (ESA) procedure due to intractable ETI failure [20]. Furthermore, it has been reported that ETI failure occurs during elective procedures in about 1 over 50,000 cases, with very high related mortality, up to $25 \%$ overall [19]. In the meta-analysis by Cabrini et al., despite preoperatively predicted difficult airway, failed ETI was reported in about $0.6 \%$ of patients who underwent an awake intubation attempt by fiberoptic guidance [21].

According to the latest guidelines of the Difficult Airway Society, when tracheal intubation cannot be accomplished, the first indication is the placement of a supraglottic non-invasive device, e.g. a laryngeal mask [22]. However, in a considerable proportion of patients these devices and subsequent face-mask ventilation fail to provide adequate oxygenation; thus, an ESA rescue procedure is required [22].

Four different ESA procedures are available so far, and the choice is strongly influenced by factors related both to the patient characteristics and to the operator preference and skills: needle cricothyrotomy (CT), scalpel $\mathrm{CT}$, percutaneous dilatational tracheotomy (PDT), and open surgical tracheotomy (OST) [23]. Technical details about OST will be reported in Section 2.2.

In case of severe hypoxemia, the fastest method to provide oxygen delivery inside the airway is a needle CT, a simple procedure that involves positioning a large-bore needle through the cricothyroid membrane and connection to an oxygen circuit. Nevertheless, needle CT should be considered a temporary solution to be followed by a definitive procedure (i.e. tracheal intubation whenever feasible, or tracheotomy) as soon as possible after the critical situation has been relieved [24]. 
Surgical or scalpel CT is the procedure of choice suggested by the Advanced Trauma Life Support (ATLS) guidelines for the establishment of an ESA [24]. The cricothyroid membrane is identified and incised with a scalpel; the incision is enlarged with a hemostat, and a tracheostomy cannula is inserted in the airway and connected to ventilation.

PDT is a multi-step guidewire-based procedure that is usually conducted under direct endoscopic control with bronchoscopy. Ciaglia first conceived this technique in 1985; since then, several kits with minor variations of the procedure have been released on the market [25]. Compared to surgical approaches, PDT allows saving time, as it is a bedside procedure technically faster than an OST, once the learning curve has been completed. The incidence of perioperative major complications and mortality is low $(8.7 \%$ and $0.3 \%$, respectively), and it is comparable to that reported in patients undergoing OST [26]. However, in the emergency setting, it is widely accepted that OST may be preferable over PDT due to the lower incidence of intraprocedural difficulties [26].

Hence, the CICO scenario is burdened by the high rate of morbidity and mortality. The establishment of preventive actions allows a reduction of such risk. Some measures have been adopted on an institutional basis, as the creation of dedicated 'difficult-airway management teams' [27, 28]. Moreover, the careful evaluation and the early identification of at-risk patients enable specifically targeted procedures to be implemented, as described by Britt and colleagues, who suggested the temporary exposure of the tracheal wall to ease the institution of an ESA at the end of neck surgery on patients with predicted difficult anatomy [29].

\section{Tracheotomy technique}

\subsection{Patient positioning and anatomical landmarks}

In the case of ETI failure, timing is critical and ideal conditions to perform a surgical tracheotomy cannot be always guaranteed [30]. However, efforts should be done to ensure conditions as similar as possible to elective tracheotomy to reduce periprocedural complications and achieve optimal results.

The patient should lie in a supine position with the head extended to expose the airway, particularly in presence of a patient with a small and stocky build. A rolled drape is placed under the scapulae to ensure appropriate extension.

The cricoid prominence and the suprasternal notch are identified as anatomical landmarks (Fig. 1A). The cricoid cartilage should be considered the referral point for the incision because the airway position is dependent on the grade of extension of the head, and the patient's build, and not on the position of the sternal notch [31].

\subsection{Procedure}

In contrast to what is suggested for elective tracheotomy, emergency OST begins with a midline vertical skin incision, starting from the cricoid and directed towards the jugular incisure of the sternum, to avoid hemorrhagic complications due to injuries of brachiocephalic structures or anterior jugular veins [30,32]. Moreover, vertical-shaped access allows better

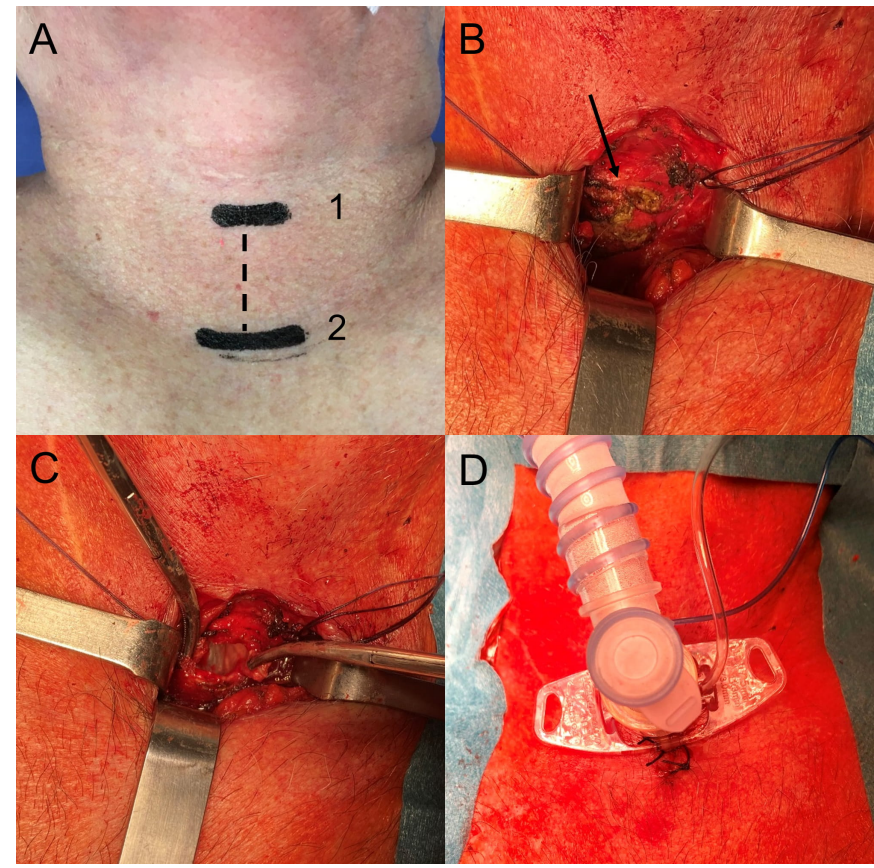

FIGURE 1. Technique of open surgical tracheotomy. Anatomical landmarks for skin incision to perform an emergency open surgical tracheotomy. (A) A vertical incision (dotted line) is created on the midline between the cricoid cartilage (1) and the sternal jugular incisure (2). (B) After thyroid isthmus division, the anterior tracheal wall is approached with an inverted ' $\mathrm{H}$ ' shaped incision (arrow) leaving intact the cricoid and the first tracheal rings. (C) The trachea is opened and (D) A tracheal cannula is inserted through the stoma. The wound is then closed around the cannula with interrupted silk stitches.

retraction of the borders of the wound and visualization of the surgical field, with the only disadvantage of worst cosmetic results once the tracheostomy has been removed [33].

The subcutaneous tissue and the platysma are dissected, and the neck strap muscles are divided on the median raphe. The thyroid isthmus is encircled, divided and the edges are sutured to expose the cricoid cartilage and the upper tracheal rings.

The pretracheal fascia is opened, the trachea is sectioned preserving the cricoid and the first tracheal rings to reduce the risk of late subglottic stenosis. In our Institute, we usually perform an inverted ' $\mathrm{H}$ ' incision between the second and the third tracheal ring (Fig. 1B); still, according to the operator preference, the tracheal wall may as well be approached with a simple vertical incision [31] or with the creation of a mucouscartilaginous flap fixed at the skin edges of the incision [32].

Once the trachea is opened (Fig. 1C), a cuffed tracheal cannula is introduced secured either with stitches to the skin or with a tape around the neck (Fig. 1D). The type of tracheal cannula is chosen based on the patient's physical constitution and the reason for the respiratory emergency. In case no tracheal cannula is available, the patient can be temporarily ventilated with an orotracheal tube placed through the tracheostoma. 


\section{ESA: should emergency tracheotomy be preferred over cricothyrotomy?}

In the past, OST was considered the procedure of choice in case of acute obstruction of the airway. In 1921 Jackson described that, in a series of 200 patients, nearly $80 \%$ of tracheal stenosis were secondary to the previous cricothyrotomy, warning against its use even in case of ESA necessity [34]. This is why conversion of emergency CT into a formal tracheostomy within 3 days has been suggested to avoid the establishment of pathologic mechanisms leading to the development of subglottic stenosis [35].

Since then, several studies later demonstrated a significant reduction in the risk of such postoperative complication [36, 37], and current ATLS [24] and DAS guidelines [22] suggest surgical CT as a rescue procedure of choice for the establishment of an ESA in the CICO scenario. Compared to OST, CT is thought to be faster and burdened by a lower incidence of intraoperative complications [38]. Moreover, emergency CT kits are widely available in both prehospital and hospital settings [22].

The incidence of perioperative complications between CT and OST in the emergency was compared in some reports. Referring to OST, higher rates of intraoperative bleeding and postoperative peristomal infections have been mentioned [39], as well as a moderately higher frequency of generic late and overall complications [37].

Nevertheless, as reported by an audit among the members of the Royal College of Anaesthetists and DAS societies in 2011, OST resulted to be the most successful rescue technique in a series of major airway-related perioperative complications; conversely, over $75 \%$ of cases managed with needle CT required a subsequent scalpel-based recovery approach because of initial failure [19]. This tendency was further confirmed by a recent systematic review by DeVore et al. [39]. The authors reviewed the existing literature about emergency CT and OST and analyzed the outcomes of the procedures: interestingly, despite the overall higher number of emergency CT reported, in centers experienced in both approaches, OST resulted in the preferred technique with a $100 \%$ success rate, while a non-negligible rate of failure in securing a safe airway was recognized as the main limitation of CT.

Therefore, still, no definitive consensus on the procedure of choice for the creation of an ESA in the case of CICO scenario has been reached so far.

Analyzing the current literature on the topic, some factors can be identified to support the choice of OST rather than CT in case of ETI failure. The first is related to anatomical factors: according to some authors, CT may result challenging in patients with destructive laryngeal traumas or with a large neck $[27,40]$, even though others suggest this approach in obese patients considering the easier detectability of the cricothyroid membrane [38].

Secondly, the risks underlying a later conversion to OST of an upfront CT ought to be carefully considered as well. In a review of studies on the outcomes of CT conversion to OST in cohorts of trauma patients, Talving and colleagues reported a high incidence of perioperative complications and mortality from 2 small previous series (53\% and $28 \%$, respectively)
[36]. In the systematic review by DeVore et al., patients who underwent conversion of an emergency CT showed longer inhospital stay [39].

Finally, conversion to formal OST cannot always be accomplished because of respiratory and hemodynamic instability, in particular when facing cardiovascular or traumatic emergencies; this would compromise the possibility to ensure a definitive airway in these categories of patients [35, 41].

Therefore, primary OST in the case of CICO scenario may be considered as the first-choice procedure in selected patients, not only in those requiring long-term ventilation. Also, this approach may prove to be time- and cost-saving with the elimination of a supplementary surgical procedure. However, this should be balanced by the associated risks. Jotic et al. identified several predictors of perioperative morbidity and mortality in patients undergoing emergency OST [42]. Attention must be paid in the case of patients that previously underwent tracheotomy or neck radiotherapy, or in those with pathologies of the neck. Higher rates of complications and mortality have been reported in cases of OST performed in contexts other than the operating room.

In conclusion, the choice between CT and OST in the case of ESA has to be driven mainly by the operator's preference and skills. Upfront OST can represent a valid choice in experienced centers in the context of institutional protocols.

\section{ESA and emergency tracheotomy in COVID-19 patients}

\subsection{ETI failure management in COVID-19 patients}

A very unstable clinical presentation is more common in patients affected by severe SARS-CoV-2-associated pneumonia, a situation that may abruptly evolve to acute respiratory failure necessitating endotracheal intubation. Since several concurrent cardiovascular and respiratory factors may enhance the possibility of difficult airway management [17, 18], a thorough evaluation of patients at risk and the establishment of preventive planned measures will reduce the occurrence of improperly demanding emergency procedures [43].

In this context, a factor that has to be considered of paramount importance is the risk of SARS-CoV-2 transmission to the healthcare staff. Following the SARS outbreak in 2002, it was demonstrated that mask ventilation before intubation, tracheotomy, and tracheal intubation lead to a three- to a sixfold increase in the risk of viral transmission to the operators [44]. Other procedures with manipulation of the airway, e.g. fiberoptic bronchoscopy, carry a significant risk of infection for the involved personnel. The early phase of the COVID-19 pandemic confirmed this trend: in fact, health professionals composed up to $28 \%$ of all cases in some states of the USA [45].

Hence, society guidelines have been recently released to specifically address the management of critical airway emergencies during the current pandemic [46-48]. Despite a univocally shared consensus on the most appropriate approach in SARS-CoV-2-positive patients has still not been defined, most authors agree that similar measures should be adopted 


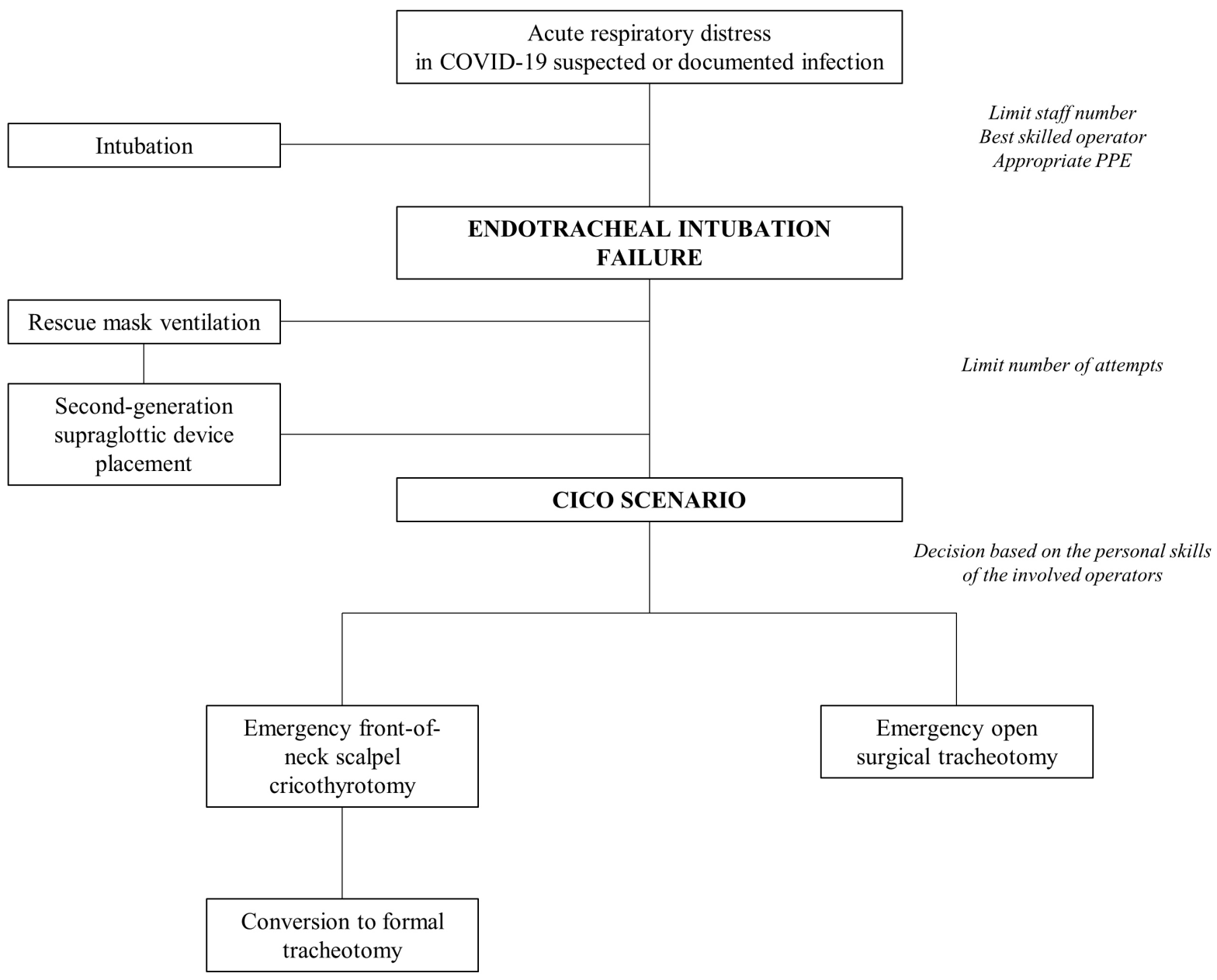

F IGURE 2. Flowchart of management of difficult airway in patients with suspected or documented SARS-CoV-2 infection.

both in the case of patients with a definitive diagnosis as well as to those with undetermined COVID-19 status [49, 50]. The primary aim of these recommendations is twofold: first, to give evidence about the most appropriate management with a systematic description of procedures that proved to be effective in the case of CICO scenario in this particular subset of patients. Second, to promote the most indicated measures to guarantee the safety of the healthcare professionals. In fact, in almost all of these guidelines, there is a large concern about the suggestion of adequate personal protective equipment (PPE), and the application of standardized measures by dedicated intubation and surgical teams composed of skilled operators [46, 51, 52].

Certainly, the risk of viral spread during airway rescue procedures is the main reason for the amendments made to traditional protocols. While some authors privilege to place a supraglottic device followed by bronchoscope-guided tracheal intubation advocating a less traumatic manipulation of respiratory mucosa $[46,49,53]$, others recommend that an emergency front-of-neck airway (eFONA) approach is preferable with acceptable rates of aerosolization if performed under protocols conditions [54]. Current indications for difficult airway management in COVID-19 patients are summarized in a flowchart (Fig. 2).

\subsubsection{Which rescue ESA technique should be recommended in COVID-19 patients?}

Following ATLS recommendations, scalpel CT is indicated as the first-choice eFONA rescue procedure by both the Safe Airway Society guidelines [46] and the consensus document released by 4 anesthesiological societies from the United Kingdom [47].

Surgical CT has certainly some advantages when compared to other techniques, because it is the most commonly trained eFONA among clinicians of both the anesthesiological and surgical fields, and it allows obtaining fast and safe airway access in both hospital and prehospital settings. Authors that support the use of scalpel CT warn against prolonged orotracheal intubation and cannula-based techniques attempts owing to the risk of viral spread because of increased airway pressures $[46,47,53]$. Nevertheless, a study from Chua et al. conducted over a manikin model refuted this hypothesis, showing that a higher amount of aerosolized components around the insertion site was encountered following surgical front-of-neck access rather than in the case of the cannula-based technique [55]. Therefore, it is still unclear if the use of scalpel CT is sufficient to guarantee an adequate safety level for healthcare workers assisting COVID-19 patients.

Although it is commonly accepted that procedures with 
TA B L E 1. Indications to elective tracheotomy in COVID-19 patients.

Traditional indications in patients with prolonged mechanical ventilation need

Specific indications in COVID-19 patients
- Favor weaning from mechanical ventilation

- Favor adequate airway toilet

- Prevent late complications (subglottic and tracheal stenosis)

- Prevent difficult reintubation

- Reduce the risk of infection of healthcare workers

- Favor early discharge from ICU

- Improve management of patients in non-intensive departments manipulation of the airway should be minimized as much as possible, some authors suggest proceeding to formal tracheotomy straight afterward a rescue airway has been obtained by surgical CT $[33,43]$. This recommendation is supported by the evidence that, once the critical situation has been relieved by emergency $\mathrm{CT}$, conversion to tracheotomy can be carried out without any pressing need, taking care of both patient and clinician safety, and thus avoiding the additional risks associated with a later staged second procedure [33].

Nevertheless, in a recent editorial, Mesolella endorsed an even more dynamic approach, suggesting upfront OST whenever ESA is needed in case of ETI failure in patients with documented or unknown SARS-CoV-2 positivity to avoid the unnecessary exposure of the involved personnel to the risk of infection carried by multiple procedures [54]. A straight OST approach was further supported by a review of guidelines and recommendations released by several organizations worldwide after the onset of the pandemic [56].

\subsection{Emergency tracheotomy: a review of the existing protocols}

Despite the magnitude of the problem of the lost airway in COVID-19 patients, international recommendations addressing OST procedure in detail are still lacking. Since the beginning of the SARS-CoV-2 outbreak, Slovenian national guidelines are to date the only ones available with specific indications for the safe management of CICO scenario with OST in patients with documented or suspected infection by novel coronavirus [33]. Nevertheless, the expertise gained during the SARS emergency in 2002 constitutes precious evidence to build up a baseline, with particular regard to healthcare workers' protection [45].

Although a common opinion on the best practice for tracheotomy performance in COVID-19 patients has still not been reached, the high risk of incidental contamination of operators during the procedure is still the most feared complication. Moreover, the emergency OST procedure has only been marginally covered by documents focusing on indications for elective tracheotomy in COVID-19 patients that require long-term mechanical ventilation (Table 1). Many authors agree that a careful application of elective treatment principles to the emergency setting allows lowering notably the risk of infection transmission to the healthcare providers [43, 46, 48, 57].

In several reports, emergency OST was employed in patients with head and neck cancers [58-60]. The unique features of these patients, besides to challenge imposed by COVIDrelated respiratory failure, require the application of tailored procedural modifications that contribute to the creation of further knowledge about the most appropriate methods for the conduction of OST procedure in SARS-CoV-2 positive patients.

Essential recommendations to perform an OST during the COVID-19 pandemic are reported in Table 2.

\subsubsection{Location and personnel involved}

Despite the higher incidence of perioperative complications of OST procedure performed in non-operating theatre context [42], most authors recommend avoiding the transport of patients between different hospital services because of the high risk of viral contamination. In those cases in which the patient's transfer appears unavoidable, reserved routes should be used. Alternatively, the transfer team could follow corridors temporarily closed to the public use [57]. In the meanwhile, the surgeons and scrub nurses should prepare wearing appropriate PPE, and get ready for patient's arrival in order to reduce the waiting time until tracheotomy procedure start [57].

Bedside OST performed in dedicated ICU was demonstrated to be feasible with no significant hazard for the patient, both in terms of perioperative and long-term complications, and for the involved clinicians [61]. Moreover, bedside procedure could be necessary as the poor respiratory and hemodynamic status caused by COVID-19 associated ARDS may hinder the transfer of the patient towards the operating room [48].

On the other hand, many authors emphasized that the risk of viral aerosolization is significantly reduced when tracheotomy is performed in negative pressure rooms [43, 46, 52, 57, 60, 62]. Certainly, this point along with the creation of dedicated routes for the transport of COVID-19 patients represents a considerable logistic and economic burden for many hospitals. During the first wave of the pandemic, our institution underwent a deep reorganization to respond to the need for health care in a highly populated metropolitan area in the north of Italy [63]. Two prefabricated tent structures previously dedicated to team sports afferent to our university were converted into 24-beds ICUs with a nearby-dedicated operating theatre to manage surgical emergencies in COVID-19 patients. This setup enabled avoiding all the risks connected with patients' movement and ensured OST to be performed in optimal conditions when required. The creation of dedicated COVID19 operating rooms is recommended by guidelines to reduce the risk of periprocedural complications of the OST that may 
TA B L E 2. Summary of recommendations for the management of emergency OST in patients with documented or unknown SARS-CoV-2 infection.

\begin{tabular}{ll}
\hline Location & - Use preferential routes to move patients to reduce the risk of contamination \\
& - Prefer dedicated negative pressure rooms to perform OST \\
& - N95 facial filter \\
& - Eye protection device \\
- Surgical hat & - Waterproof gown \\
& - Double gloves
\end{tabular}

- Prefer general anesthesia with neuromuscular blockade to reduce the risk of Anesthesia and ventilation management aerosol generation

- Preoxigenation via face mask or supraglottic device 3-5 minutes

- Hold ventilation before tracheal wall incision

- Prefer immediate conversion of emergency scalpel CT to formal OST or directly perform OST to avoid the risks carried by a staged procedure

Technical modifications

- Consider H-shaped tracheal wall incision to ease later substitution of tracheal cannula

- Avoid suction, or use closed suction systems

- Cold hemostasis technique, avoid electrocautery

- Verify the position of the cannula with end-tidal $\mathrm{CO} 2$ evaluation

be prohibitive if performed bedside in patients with difficult anatomy [33].

Most studies suggest that the choice of ESA technique should depend ultimately on personal operator preference based on his or her own experience. In general, OST approach is recommended in the presence of well-trained ENT specialists or thoracic surgeons [64]. Whenever possible, surgical staff should be alerted in case of a predicted difficult airway if a PDT attempt is decided [43, 48]. However, in the context of the current pandemic, surgical skills widening to a larger audience could be of help. Picetti et al. reported that OST could be performed with success and low complication rate by intensivists trained with the technique [61].

\subsubsection{PPE}

The adoption of appropriate PPE devices is the most important aspect to be considered in the management of emergency OST. Several studies demonstrated that the risk of SARS-CoV-2 transmission to healthcare workers is negligible if standardized protective measures are employed [33, 61].

The use of several types of facial filters has been reported by the studies exploring the management of CICO scenario in COVID-19 patients. Some authors suggest that, in the case of aerosol-producing procedures like OST, operators should use powered air-purifying respirators (PAPRs) [33, 52, 62]. However, some uncertainties with this indication arise because of their limited availability, the long time required to wear them - not always compatible with emergencies - and the generation of noise that could interfere with communications between the members of the emergency team $[33,61]$.

Protection from droplets and aerosols generated in the course of OST was provided with N95 masks in most of the studies present in the literature. Among PPE, this device is generally considered as a baseline ensuring an acceptable balance between healthcare personnel safety, availability, and costs [33, 43, 46, 50, 52, 62]. Considering the difficulties related to the continuous supply of PPE in some areas, it could be advisable to wear a surgical mask over the N95 respirator to prevent contamination of the device during its period of activity [62].

Besides facial filters, basic PPE to prevent airborne infections should also include protective eyewear, a surgical cap, a water-resistant gown, double gloves, and shoe covers $[52,57,62]$.

\subsubsection{Anesthetic technique}

Anesthesiological management of COVID-19 patients undergoing OST ranges from awake procedures to general anesthesia. Even though full sedation and neuromuscular blockade are preferable to minimize the periprocedural generation of aerosols due to cough reflex [33, 43, 46, 48, 57], this approach may not be viable in particular subsets of patients, including the trauma population and those affected by unsolvable airway obstructions caused by head and neck masses [50, 58, 59].

Ahmad and colleagues reported a case of awake OST following difficult tracheal intubation in a patient presenting with airway obstruction caused by a tumor of the tongue and suspected SARS-CoV-2 infection [58]. The case was managed with conscious sedation by target-controlled infusion of propofol and remifentanil along with pharyngeal topical anesthesia administration to prevent cough reflex during bronchoscopyguided intubation; following intubation, OST was performed under safe conditions in general anesthesia and curarization.

OST under conscious sedation was accomplished in another 
patient with a transglottic mass impeding preoperative tracheal intubation who was ventilated through a filtered mask accurately secured to the face to prevent air leaks during the procedure [59]. A similar approach was described by Hassani et al. during the current pandemic in two patients with facial and airway trauma necessitating OST because of intubation inability [50]. The awake OST airway was only successful in one case, administrating local lidocaine. In the second case, additional intravenous sedation was required because of psychomotor restlessness. In both cases, muscle relaxation was induced once a secure airway had been obtained.

Most authors agree that adequate preoxygenation should be delivered to the patients before beginning OST procedure to prevent the early onset of acute respiratory failure [46]. Oxygen can be supplemented via a nasal cannula or a tightly secured face mask, but high-flow devices should be avoided because of the increased risk of aerosolization [46, 53]. The optimal duration of preoxygenation ranges between 3 to 5 minutes $[33,46]$.

Chen and colleagues reported an interesting case of a Chinese patient who underwent emergency OST for acute airway obstruction caused by an advanced stage laryngeal tumor during the SARS-CoV-2 outbreak [65]. Despite the emergency setting and contraindication to tracheal intubation, the patient was supported with veno-venous extracorporeal membrane oxygenation (ECMO) to prevent periprocedural severe hypoxia, allowing proper planning of OST procedure and recommended safety maintenance.

\subsubsection{Technical considerations}

When the procedure is required in patients with documented or suspected infection by the novel coronavirus, general principles of emergency OST should be followed. Nevertheless, several modifications have been documented by the studies reporting experience in the field, with the primary aim of diminishing the risk of transmission to healthcare operators during surgery.

It is recommended that both neck dissection and hemostasis phases should be conducted using cold instruments. Cauterization increases the risk of aerosol generation and should be avoided whenever possible [33, 52]. Manual compression of bleeding sources is in most cases adequate to provide excellent hemostasis at the time of dissection [52]. Nevertheless, Broderick et al. underline the important role of diathermic instruments for careful hemostasis, in the light of the high incidence of coagulation alterations reported in COVID-19 patients. According to the authors, the generation of aerosols can be lowered with cautious suction of generated smokes [57].

Whether formal tracheal intubation is possible or the patient receives oxygenation through a face mask or nasal cannula, almost all authors recommend that ventilation should be held at the time of tracheal wall incision to avoid the dangerous spreading of air particles [33, 48, 52, 53, 57, 62]. The endotracheal tube, if present, should be retracted proximally to the tracheal incision site [58], or advanced at the level of the main carina without deflating the cuff to prevent its accidental damage and spreading [33, 57].

Suction during dissection and following tracheal opening is considered a procedure with a high risk of aerosol generation and should be excluded [62]; nevertheless, a closed suction system could be carefully used if necessary $[52,60]$.

Pronation has been demonstrated to be useful in COVID19 patients in increasing gas exchange in the acute phase of viral pneumonia. However, a prone position augments the risk of tracheal cannula dislodgement, and may hinder a quick replacement of the device, in particular in patients who underwent PDT with a tight tracheostoma [64]. On the other hand, the creation of a tracheal flap is a longer procedure that leads to the creation of a wide interface between the airway and the surrounding environment with a high risk of airborne infections [64]. Accordingly, the inverted ' $\mathrm{H}$ ' shaped tracheal wall incision technique, as adopted in our Institution, may represent a good compromise to ensure proper safety for both the patient and the operators.

Finally, after tracheal incision and tracheal cannula positioning, the correct introduction of the device should be checked after inflating the cuff, and possibly with end-tidal $\mathrm{CO}_{2}$ evaluation to avoid the risk of aerosol generation in case of bronchoscopy [53, 57, 58, 60].

\section{Conclusions}

The occurrence of the CICO scenario in patients affected by COVID-19-related ARDS represents a life-threatening event. Despite the lack of current international dedicated guidelines, evidence on the best approach to this emergency can be inferred by traditional protocols implemented by specific modifications to ensure the acceptable risk of infection for the healthcare workers. OST may represent an appropriate ESA in the case of patients with facial trauma and/or head and neck cancers. Moreover, upfront OST allows avoiding additional conversion of temporary CT. Particular caution must be taken considering the use of adequate PPE and procedural modifications to reduce the rate of aerosol generation during OST.

\section{Author contributions}

PM and PC conceived the study. PM, PC and AC performed the literature search and analyzed the data. PM wrote the manuscript. PC and GN revised the original version of the manuscript. All authors contributed to editorial changes in the manuscript. All authors read and approved the final manuscript.

\section{Acknowledgment}

Thanks to all the peer reviewers for their opinions and suggestions.

\section{Funding}

No external funding received.

\section{Conflict of interest}

The authors declare no competing interests. 


\section{REFERENCES}

[1] Zhu N, Zhang D, Wang W, Li X, Yang B, Song J, et al. A novel coronavirus from patients with pneumonia in China, 2019. New England Journal of Medicine. 2020; 382: 727-733.

[2] Salzberger B, Buder F, Lampl B, Ehrenstein B, Hitzenbichler F, Holzmann T, et al. Epidemiology of SARS-CoV-2. Infection. 2020; 17.

[3] World Health Organization. WHO announces COVID-19 outbreak a pandemic. Accessible at: https://ww. euro.who.int/en/ health-topics/health-emergencies/coronavirus-covid19/news/news/2020/3/who-announces-covid-19-outbreaka-pandemic

[4] Berlin DA, Gulick RM, Martinez FJ. Severe COVID-19. New England Journal of Medicine. 2020; 383: 2451-2460.

[5] Kumar R, Srivastava JK, Singh R, Siddiqui MH, Mansouri RA, Abdulhakim JA, et al. Available compounds with therapeutic potential against COVID-19: antimicrobial therapies, supportive care, and probable vaccines. Frontiers in Pharmacology. 2020; 11: 582025.

[6] Polack FP, Thomas SJ, Kitchin N, Absalon J, Gurtman A, Lockhart S, et al. Safety and efficacy of the BNT162b2 mRNA COVID-19 vaccine. New England Journal of Medicine. 2020; 383: 2603-2615.

[7] Karaba SM, Jones G, Helsel T, Smith LL, Avery R, Dzintars K, et al. Prevalence of co-infection at the time of hospital admission in COVID19 patients, a multicenter study. Open Forum Infectious Diseases. 2020; 8: ofaa578.

[8] Mahmoudi H. Bacterial co-infections and antibiotic resistance in patients with COVID-19. GMS Hygiene and Infection Control. 2020; 15: Doc35.

[9] Cataldo MA, Tetaj N, Selleri M, Marchioni L, Capone A, Caraffa E, et al. Incidence of bacterial and fungal bloodstream infections in COVID19 patients in intensive care: an alarming "collateral effect". Journal of Global Antimicrobial Resistance. 2020; 23: 290-291.

[10] Gattinoni L, Chiumello D, Caironi P, Busana M, Romitti F, Brazzi L, et al. COVID-19 pneumonia: different respiratory treatments for different phenotypes? Intensive Care Medicine. 2020; 46: 1099-1102.

[11] Grieco DL, Bongiovanni F, Chen L, Menga LS, Cutuli SL, Pintaudi G, et al. Respiratory physiology of COVID-19-induced respiratory failure compared to ARDS of other etiologies. Critical Care. 2020; 24: 529.

[12] Balzanelli M, Distratis P, Catucci O, Amatulli F, Cefalo A, Lazzaro R, et al. Clinical and diagnostic findings in COVID-19 patients: an original research from SG Moscati Hospital in Taranto Italy. Journal of Biological Regulators and Homeostatic Agents. 2021; 35: 171-183.

[13] Turcato G, Panebianco L, Zaboli A, Scheurer C, Ausserhofer D, Wieser A, et al. Correlation between arterial blood gas and CT volumetry in patients with SARS-CoV-2 in the emergency department. International Journal of Infectious Diseases. 2020; 97: 233-235.

[14] Shang Y, Xu C, Jiang F, Huang R, Li Y, Zhou Y, et al. Clinical characteristics and changes of chest $\mathrm{CT}$ features in 307 patients with common COVID-19 pneumonia infected SARS-CoV-2: a multicenter study in Jiangsu, China. International Journal of Infectious Diseases. 2020; 96: 157-162.

[15] Berlin DA, Gulick RM, Martinez FJ. Severe COVID-19. New England Journal of Medicine. 2020; 383: 2451-2460.

[16] Lentz S, Roginski MA, Montrief T, Ramzy M, Gottlieb M, Long B. Initial emergency department mechanical ventilation strategies for COVID19 hypoxemic respiratory failure and ARDS. The American Journal of Emergency Medicine. 2020; 38: 2194-2202.

[17] Zheng H, Li S, Sun R, Yang H, Chi X, Chen M, et al. Clinical experience with emergency endotracheal intubation in COVID-19 patients in the intensive care units: a single-centered, retrospective, descriptive study. American Journal of Translational Research. 2020; 12: 6655-6664.

[18] Yao W, Wang T, Jiang B, Gao F, Wang L, Zheng H, et al. Emergency tracheal intubation in 202 patients with COVID-19 in Wuhan, China: lessons learnt and international expert recommendations. British Journal of Anaesthesia. 2020; 125: e28-e37.

[19] Potnuru P, Artime CA, Hagberg CA. The lost airway. Anesthesiology Clinics. 2020; 38: 875-888.

[20] Ono Y, Kakamu T, Kikuchi H, Mori Y, Watanabe Y, Shinohara $\mathrm{K}$. Expert-performed endotracheal intubation-related complications in trauma patients: incidence, possible risk factors, and outcomes in the prehospital setting and emergency department. Emergency Medicine International. 2018; 2018: 5649476.

[21] Cabrini L, Baiardo Redaelli M, Ball L, Filippini M, Fominskiy E, Pintaudi $\mathrm{M}$, et al. Awake fiberoptic intubation protocols in the operating room for anticipated difficult airway: a systematic review and meta-analysis of randomized controlled trials. Anesthesia \& Analgesia. 2019; 128: 971980.

[22] Frerk C, Mitchell VS, McNarry AF, Mendonca C, Bhagrath R, Patel A, et al. Difficult Airway Society 2015 guidelines for management of unanticipated difficult intubation in adults. British Journal of Anaesthesia. 2015; 115: 827-848.

[23] Patel SA, Meyer TK. Surgical airway. International Journal of Critical Illness and Injury Science. 2014; 4: 71-76.

[24] Airway and ventilatory management. In Henry S, Brasel K, Stewart RM, editors. ATLS, Advanced Trauma Life Support, Student course manual (pp. 22-41). 10th edn. Chicago, IL: American College of Surgeons. 2018.

[25] Sheykholeslami K, Rezaee R, Lavertu P. Percutaneous tracheotomy. Atlas of the oral and maxillofacial surgery clinics of North America. 2010; 18 : 51-60.

[26] Klotz R, Probst P, Deininger M, Klaiber U, Grummich K, Diener MK, et al. Percutaneous versus surgical strategy for tracheostomy: a systematic review and meta-analysis of perioperative and postoperative complications. Langenbeck's Archives of Surgery. 2018; 403: 137-149.

[27] Gillespie MB, Eisele DW. Outcomes of emergency surgical airway procedures in a hospital-wide setting. The Laryngoscope. 1999; 109: 1766-1769.

[28] Darby JM, Halenda G, Chou C, Quinlan JJ, Alarcon LH, Simmons RL. Emergency surgical airways following activation of a difficult airway management team in hospitalized critically ill patients: a case series. Journal of Intensive Care Medicine. 2018; 33: 517-526.

[29] Britt CJ, Rohrbach MR, McCulloch TM. Tracheal exposure: anticipatory management of the difficult airway. Head \& Neck. 2016; 38: E2446E2448.

[30] Abdelkader M, Dempster J. Emergency tracheostomy: indications and technique. Surgery. 2003; 21: 153-155.

[31] Grillo HC. Surgery of the trachea and bronchi. Der Chirurg: Zeitschrift Fur Alle Gebiete Der Operativen Medizen. 1987; 58: 511-520. (In German)

[32] Taylor CB, Otto RA. Open tracheostomy procedure. Atlas of the Oral and Maxillofacial Surgery Clinics of North America. 2015; 23: 117-124.

[33] Šifrer R, Urbančič J, Piazza C, van Weert S, García-Purriños F, Benedik $\mathrm{J}$, et al. Emergent tracheostomy during the pandemic of COVID-19: Slovenian National recommendations. European Archives of Oto-RhinoLaryngology. 2020; 1-9.

[34] Jackson C. High tracheotomy and other errors-the chief causes of chronic laryngeal stenosis. The American Journal of the Medical Sciences. 1921; 32: 392-398.

[35] Macêdo MB, Guimarães RB, Ribeiro SM, Sousa KMMD. Emergency cricothyrotomy: temporary measure or definitive airway? A systematic review. Revista do Colegio Brasileiro De Cirurgioes. 2016; 43: 493-499.

[36] Talving P, DuBose J, Inaba K, Demetriades D. Conversion of emergent cricothyrotomy to tracheotomy in trauma patients. Archives of Surgery. 2010; 145: 87-91.

[37] Zasso FB, You-Ten KE, Ryu M, Losyeva K, Tanwani J, Siddiqui N. Complications of cricothyroidotomy versus tracheostomy in emergency surgical airway management: a systematic review. BMC Anesthesiology. 2020; 20: 216.

[38] Dillon JK, Christensen B, Fairbanks T, Jurkovich G, Moe KS. The emergent surgical airway: cricothyrotomy vs. tracheotomy. International Journal of Oral and Maxillofacial Surgery. 2013; 42: 204-208.

[39] DeVore EK, Redmann A, Howell R, Khosla S. Best practices for emergency surgical airway: a systematic review. Laryngoscope Investigative Otolaryngology. 2019; 4: 602-608.

[40] Sadda R, Turner M. Emergency tracheotomy in the dental office. International Journal of Oral and Maxillofacial Surgery. 2009; 38: 1114 1115 .

[41] Warner MA, Smith HM, Zielinski MD. Impaired ventilation and oxygenation after emergency cricothyrotomy: recommendations for the management of suboptimal invasive airway access. A \& A Case Reports. 2016; $7:$ 212-214. 
[42] Jotic AD, Milovanovic JP, Trivic AS, Folic MM, Krejovic-Trivic SB, Radin ZZ, et al. Predictors of complications occurrence associated with emergency surgical tracheotomy. Otolaryngology-Head and Neck Surgery. 2021; 164: 346-352.

[43] Radhakrishnan S, Perumbally HA, Surya S, Ponneth MS. Guidelines for surgical tracheostomy and tracheostomy tube change during the COVID19 pandemic: a review article. Indian Journal of Otolaryngology and Head \& Neck Surgery. 2020; 72: 398-401.

[44] Tran K, Cimon K, Severn M, Pessoa-Silva CL, Conly J. Aerosol generating procedures and risk of transmission of acute respiratory infections to healthcare workers: a systematic review. PLoS ONE. 2012; 7: e35797.

[45] Skoog H, Withrow K, Jeyarajan H, Greene B, Batra H, Cox D, et al. Tracheotomy in the SARS-CoV-2 pandemic. Head \& Neck. 2020; 42: 1392-1396.

[46] Brewster DJ, Chrimes N, Do TB, Fraser K, Groombridge CJ, Higgs A, et al. Consensus statement: Safe Airway Society principles of airway management and tracheal intubation specific to the COVID-19 adult patient group. Medical Journal of Australia. 2020; 212: 472-481.

[47] Cook TM, El-Boghdadly K, McGuire B, McNarry AF, Patel A, Higgs A. Consensus guidelines for managing the airway in patients with COVID19: guidelines from the difficult airway society, the Association of Anaesthetists the Intensive Care Society, the Faculty of Intensive Care Medicine and the Royal College of Anaesthetists. Anaesthesia. 2020; 75: 785-799.

[48] Michetti CP, Burlew CC, Bulger EM, Davis KA, Spain DA. Performing tracheostomy during the COVID-19 pandemic: guidance and recommendations from the Critical Care and Acute Care Surgery Committees of the American Association for the Surgery of Trauma. Trauma Surgery \& Acute Care Open. 2020; 5: e000482.

[49] Shiba T, Ghazizadeh S, Chhetri D, St. John M, Long J. Tracheostomy Considerations during the COVID-19 Pandemic. OTO Open. 2020; 4: 2473974 X20922528.

[50] Hassani V, Amniati S, Ahmadi A, Mohseni M, Sehat-Kashani S, Nikoubakht N, et al. Emergency tracheostomy in two airway trauma patients suspected of COVID-19: a case report. Anesthesia and Pain Medicine. 2020; 10: e104648.

[51] Ahmad I, Jeyarajah J, Nair G, Ragbourne SC, Vowles B, Wong DJN, et al. A prospective, observational, cohort study of airway management of patients with COVID-19 by specialist tracheal intubation teams. Canadian Journal of Anesthesia. 2021; 68: 196-203.

[52] Lee D, Kim S, Kim J, Kim BG, Chang K, Park J. Protection of medical staff during tracheotomy: lessons learned from the COVID-19 pandemic. Journal for Oto-Rhino-Laryngology, Head and Neck Surgery. 2020; 82: 304-309.

[53] Lima DS, Ribeiro Junior MF, Vieira-Jr HM, Campos T, Saverio SD Alternatives for establishing a surgical airway during the COVID19 pandemic. Revista do Colégio Brasileiro de Cirurgiões. 2020; 47: e20202549.

[54] Mesolella M. Is timing of tracheotomy a factor influencing the clinical course in COVID-19 patients? Ear, Nose \& Throat Journal. 2021; 100: 120S-121S.

[55] Chua H, Wong T, Lim WY, Wong P. Emergency front-of-neck airway in the COVID-19 patient: Cannula or surgical cricothyroidotomy? Anaesthesia Critical Care \& Pain Medicine. 2020; 39: 475-477.

[56] Heyd CP, Desiato VM, Nguyen SA, O’Rourke AK, Clemmens CS, Awad MI, et al. Tracheostomy protocols during COVID-19 pandemic. Head \& Neck. 2020; 42: 1297-1302.

[57] Broderick D, Kyzas P, Sanders K, Sawyerr A, Katre C, Vassiliou L. Surgical tracheostomies in COVID-19 patients: important considerations and the " 5 Ts" of safety. British Journal of Oral and Maxillofacial Surgery. 2020; 58: 585-589.

[58] Ahmad I, Wade S, Langdon A, Chamarette H, Walsh M, Surda P. Awake tracheal intubation in a suspected COVID-19 patient with critical airway obstruction. Anesthesiology Reports. 2020; 8: 28-31.

[59] Crossley J, Clark C, Brody F, Maxwell JH. Surgical considerations for an awake tracheotomy during the COVID-19 pandemic. Journal of Laparoendoscopic \& Advanced Surgical Techniques. 2020; 30: 477-480.

[60] George CK, Varghese BT, Divya GM, Janardhan D, Thomas S. Emergency tracheostomy during COVID 19 pandemic in a head and neck surgical oncology unit. Oral Oncology. 2020; 107: 104784.

[61] Picetti E, Fornaciari A, Taccone FS, Malchiodi L, Grossi S, Di Lella F, et al. Safety of bedside surgical tracheostomy during COVID-19 pandemic: A retrospective observational study. PLoS ONE. 2020; 15: e0240014.

[62] Bier-Laning C, Cramer JD, Roy S, Palmieri PA, Amin A, Añon JM, et al. Tracheostomy during the COVID-19 pandemic: comparison of international perioperative care protocols and practices in 26 countries. Otolaryngology-Head and Neck Surgery. 2020; 194599820961985.

[63] Zangrillo A, Beretta L, Silvani P, Colombo S, Scandroglio AM, Dell'Acqua A, et al. Fast reshaping of intensive care unit facilities in a large metropolitan hospital in Milan, Italy: facing the COVID-19 pandemic emergency. Critical Care and Resuscitation. 2020; 22: 91-94.

[64] Botti C, Lusetti F, Neri T, Peroni S, Castellucci A, Salsi P, et al. Comparison of percutaneous dilatational tracheotomy versus open surgical technique in severe COVID-19: complication rates, relative risks and benefits. Auris Nasus Larynx. 2020; S0385-8146(20)30296-0.

[65] Chen Z, Lv Y, Feng Y. Case report of acute airway obstruction caused by transglottic squamous carcinoma (stage IV) during the coronavirus pandemic cured by ECMO-assisted tracheostomy. Ear, Nose \& Throat Journal. 2021; 100: 113S-115S.

How to cite this article: Piergiorgio Muriana, Paola Ciriaco, Angelo Carretta, Giampiero Negri. Emergency surgical tracheotomy in the era of COVID-19 pandemic. Signa Vitae. 2021;17(3):6978. doi:10.22514/sv.2021.055. 